\title{
Development of particle migration in pressure-driven flow of a Brownian suspension
}

\author{
DENIS SEMWOGERERE ${ }^{1}$, JEFFREY F. MORRIS \\ AND ERIC R. WEEKS ${ }^{1}$ \\ ${ }^{1}$ Physics Department, Emory University, Atlanta, GA 30322, USA \\ ${ }^{2}$ Benjamin Levich Institute and Department of Chemical Engineering, City College of New York, \\ New York, NY 10031, USA
}

(Received 30 August 2006 and in revised form 10 January 2007)

An experimental investigation into the influence of Brownian motion on shear-induced particle migration of monodisperse suspensions of micrometre-sized colloidal particles is presented. The suspension is pumped through a $50 \mu \mathrm{m} \times 500 \mu \mathrm{m}$ rectangular crosssection glass channel. The experiments are characterized chiefly by the sample volume fraction $(\phi=0.1-0.4)$, and the flow rate expressed as the Péclet number $(P e=10-$ 400). For each experiment we measure the entrance length, which is the distance from the inlet of the channel required for the concentration profile to develop to its non-uniform steady state. The entrance length increases strongly with increasing $P e$ for $P e \ll 100$, in marked contrast to non-Brownian flows for which the entrance length is flow-rate independent. For larger $P e$, the entrance length reaches a constant value which depends on the other experimental parameters. Additionally, the entrance length decreases with increasing $\phi$; this effect is strongest for low $\phi$. Modelling of the migration based on spatial variation of the normal stresses due to the particles captures the primary features observed in the axial evolution over a range of $P e$ and $\phi$.

\section{Introduction}

The pressure-driven flow of concentrated suspensions through a channel leads to shear-induced migration of the particle phase relative to the suspending fluid. For flows through a narrow channel, an initially uniform suspension becomes less concentrated near the walls and more concentrated near the centre of the channel. This can lead to effects such as a modification of the velocity profile of the suspension (Lyon \& Leal 1998) and a change in the system pressure drop (Miller \& Morris 2006). Shear-induced particle migration is relevant to applications where transfer of a suspension from one vessel to another is required. Thus, it impacts industries such as paper coating and food processing. Particle migration known as the FahreusLindqvist effect is also observed in blood flow (Fournier 1999).

Particle migration in suspension flows has been the subject of simulation and modelling studies (Nott \& Brady 1994; Morris \& Brady 1998) as well as experimental work (Koh, Hookham \& Leal 1994; Lyon \& Leal 1998). Particle migration has been much studied beginning with Leighton \& Acrivos (1987), but there has been little work on suspensions of particles for which Brownian motion is significant. The latter case will become increasingly important as the number of microfluidic applications increases. For Brownian suspensions, thermally driven (Brownian) 
stresses on the particles can have a significant influence on the cross-stream migration, as observed experimentally and in modelling by Frank et al. (2003). The gradient diffusion resulting from Brownian motion has the effect of opposing the migration caused by the shear-driven stresses. The influence of diffusion diminishes relative to hydrodynamically driven migration as the flow rate is increased because shear-driven stresses increase in magnitude, while thermally driven stresses remain unchanged. In pressure-driven flow, the migration thus leads to larger concentrations in the low-shear regions near the centre of the channel for larger flow rates. This flow-ratedependent extent of migration is a fundamental difference between Brownian and non-Brownian suspensions. Frank et al. (2003) focused on the study of fully evolved concentration profiles of a Brownian suspension flowing over a range of $P e$. They investigated a single monodisperse suspension at three different volume fractions and assumed the experimental data had reached full development. In this paper, we report measurements of the axial evolution of the particle concentration profile of monodisperse suspensions of Brownian particles of two different sizes $-2.3 \mu \mathrm{m}$ and $1.4 \mu \mathrm{m}$ in diameter - pumped through a $50 \mu \mathrm{m} \times 500 \mu \mathrm{m}$ cross-section microchannel, providing results reliably to values as low as $P e \approx 10$. Because the steepest variation with respect to $P e$ occurs at $P e=O(10)-O(100)$ for the concentrations studied, this is crucial to understanding the evolution dependence on the flow rate. Our studies demonstrate that in contrast with non-Brownian suspensions, flow rate has a significant effect on the development of the concentration profile in a Brownian suspension, and establishes that $P e$ determines the transition from strongly Brownian to weakly Brownian behaviour. We also show experimentally and numerically a strong dependence of the concentration development on volume fraction.

\section{Experimental methods}

Two sets of colloidal suspension are used. One is similar to that of Frank et al. (2003) and consists of $2.3 \mu \mathrm{m}$-diameter slightly charged poly-methylmethacrylate hard spheres suspended in a cyclohexylbromide/decalin mixture. The solvent matches the density of the colloidal particles to prevent sedimentation, and also matches the index of refraction to allow for visualization deep into the suspension. The other set is a similar suspension of $1.4 \mu \mathrm{m}$-diameter spherical particles. To avoid aggregation due to van der Waals attraction, the spheres are sterically stabilized by a thin layer of poly-12-hydroxystearic acid (Antl et al. 1986). In addition, the particles are labelled with fluorescent rhodamine dye to enable visualization with laser scanning confocal microscopy.

The flow chamber of figure $1(b)$ is a glass channel $10 \mathrm{~cm}$ in axial length with a $50 \mu \mathrm{m} \times 500 \mu \mathrm{m}$ rectangular cross-section (Friedrich and Dimmock). It is the same type as that used in Frank et al. (2003), but double the length. However, the plumbing connecting the syringe pump to the flow chamber has been substantially modified in order to provide a clean entrance condition at the opening of the flow chamber: the chamber is glued inside a $900 \mu \mathrm{m} \times 900 \mu \mathrm{m}$ glass channel which is itself connected on either side to a Teflon tube; the glue seals around the smaller flow chamber so that the entire flow is directed through the $50 \mu \mathrm{m} \times 500 \mu \mathrm{m}$ chamber. The larger square channel allows visual access with a microscope to the entire flow chamber; most importantly, to the chamber entrance and first few hundred micrometres.

Data is acquired using a fast confocal microscope (VT-Eye by VisiTech International) to image hundreds of particles and resolve them even when they are flowing at high speed. With a field of view of $55 \times 55 \mu \mathrm{m}^{2}$ when using a $100 \times, 1.35$ 

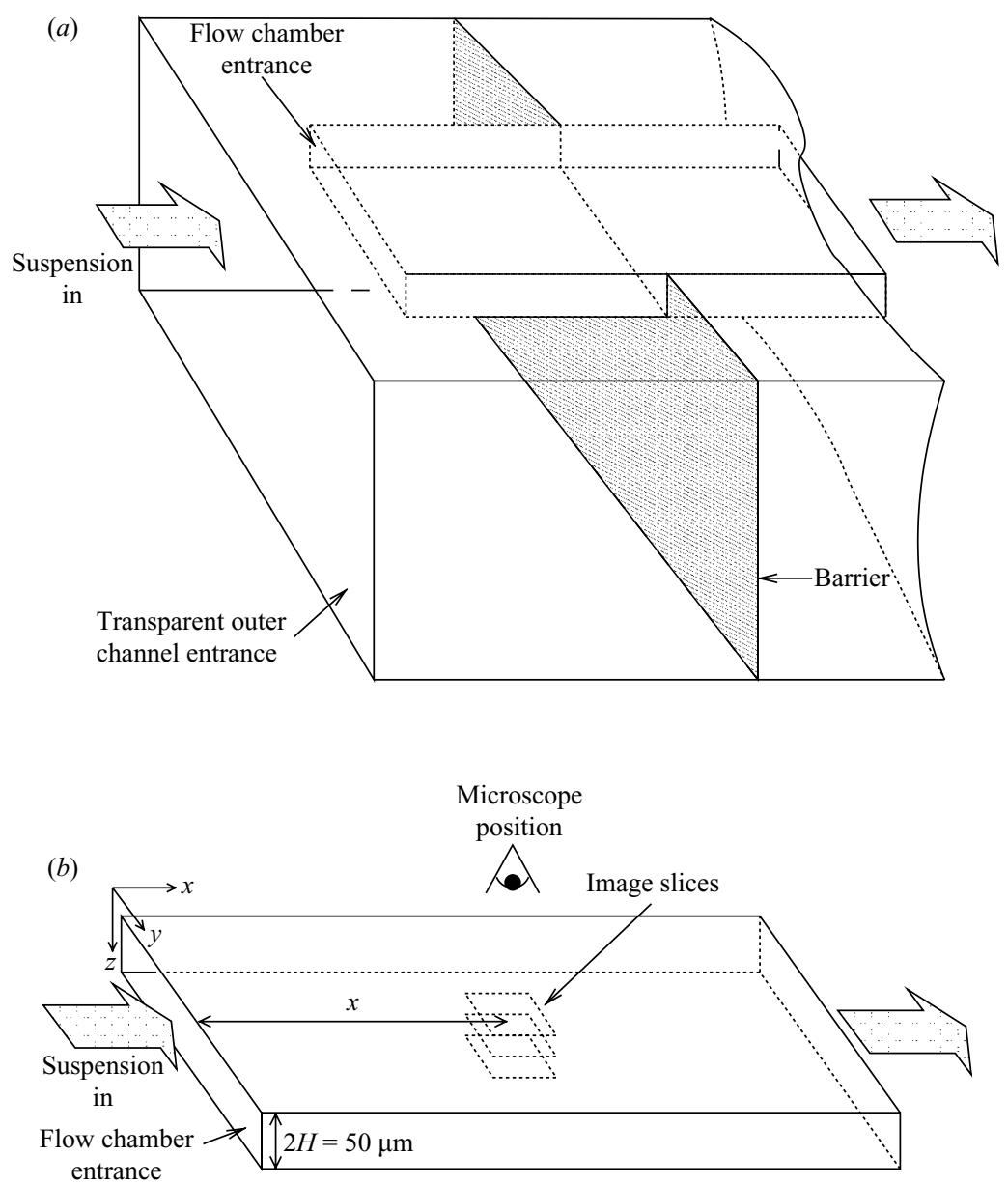

FiguRE 1. (a) Schematic of the experimental set-up at the entrance of the rectangular cross-section flow chamber (capillary tube). The flow chamber is placed inside a larger rectangular cross-section glass tube which is itself connected to the Teflon tube that is attached to the syringe containing colloids. The arrangement makes the entrance of the flow chamber optically accessible to the confocal microscope. (b) Flow chamber schematic showing relative position of the microscope and the corresponding image slices. Note that length of capillary tube is not shown to scale (it is $4000 H$ ).

N.A. objective, the confocal microscope acquires images at 94 frames per second and can thus resolve particles flowing at up to $5000 \mu \mathrm{m} \mathrm{s}^{-1}$ - for these experiments, the maximum particle speeds reach $O(1000) \mu \mathrm{m} \mathrm{s}^{-1}$.

Data is collected at various points along the flow axis ( $x$-axis figure 1$)$ of the channel. The first point is at the entrance of the tube $(x=0)$ : the microscope objective is positioned to focus just inside the entrance and a series of two-dimensional slices along the $z$-axis (figure 1) is taken through the depth of the rectangular-shaped channel, i.e. from $z=0$ to $z=50 \mu \mathrm{m}$. Figure 2 shows three such image slices taken at different $z$ positions where the flow speed ranges from nearly stationary up to $430 \mu \mathrm{m} \mathrm{s}^{-1}$. From the stacks of images, the average local volume fraction along the $z$-axis is extracted by counting the number of particles observed in each image slice. This concentration profile, $\phi(x, z)$, is shown in figure 3 , where data taken at different values of $x$ reveal the axial development of the profile. 

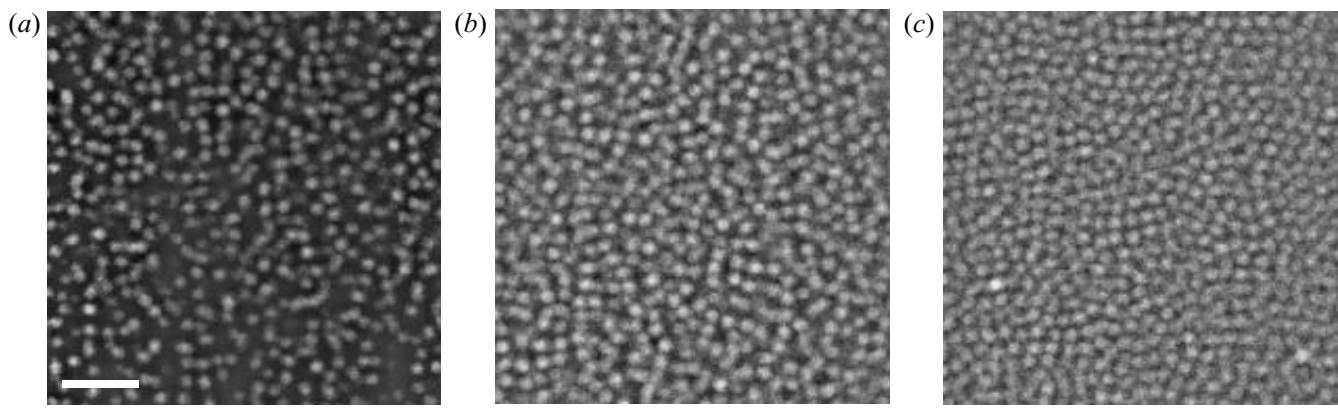

FIGURE 2. Two-dimensional slices, taken along the vertical axis $(z)$, of $2.3 \mu \mathrm{m}$ diameter particles flowing through the channel at distance $x / H=240$ from the entrance. $H=25 \mu \mathrm{m}$ is half of the channel width. The slices are at $(a) z=3 \mu \mathrm{m},(b) z=13 \mu \mathrm{m}$, and $(c) z=28 \mu \mathrm{m}$, where $z=0$ and $z=25$ are, respectively, the positions of one wall and the centre of the channel. The suspension is of bulk solids volume fraction 0.35 and flows at $P e=295$, corresponding to volume flow rate of $7 \mathrm{nl} \mathrm{s}^{-1}$ and $u_{\max }=295 \mu \mathrm{m} \mathrm{s}^{-1}$. Particle migration is nearly fully developed and is visible by eye: the slice closest to the wall $(z=3 \mu \mathrm{m}, z / H=0.1)$ clearly has fewer particles than the slice closest to the centre $(z=28 \mu \mathrm{m}, z / H=1.1)$. The scale bar is $10 \mu \mathrm{m}$ in length.

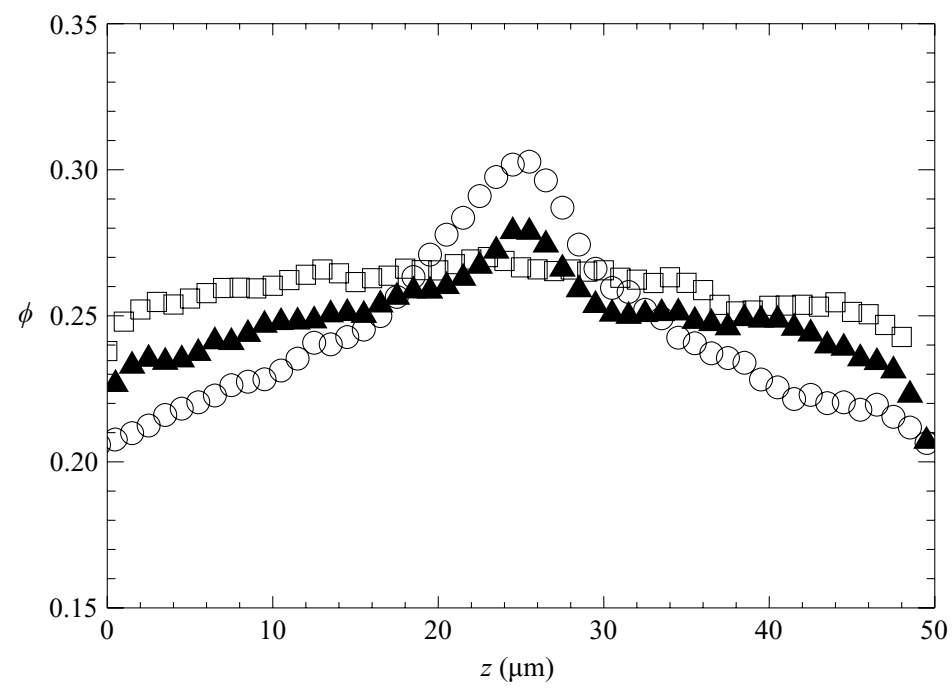

FIGURE 3. Volume fraction profile at three different positions along the channel for a suspension of $1.4 \mu \mathrm{m}$ diameter particles at volume fraction $\phi=0.26$ and $P e=129$ (volume flow rate $17 \mathrm{nl} \mathrm{s}^{-1}$ ). The volume fraction at the centre is clearly increasing with distance from the entrance. $\square$, profile at the entrance; $\boldsymbol{\Lambda}$, profile at $x / H=80 ; \bigcirc$, profile at $x / H=1360$.

The bulk volume fraction $\phi_{\text {bulk }}$ of the suspension is measured with the flow turned off, and then by counting and adding up the number of particles in each slice of a stack of two-dimensional images. On average, the total number, $N$, of particles counted in the stack of images is larger than the actual number of particles by a factor $\sim 2 a / \Delta z$, where $\Delta z$ is the separation between slices and $a$ is the particle radius. Thus,

$$
\phi_{\text {bulk }}=\frac{(N /(2 a / \Delta z)) \frac{4}{3} \pi a^{3}}{L_{x} L_{y} L_{z}},
$$

where $L_{x}$ and $L_{y}$ are the length and width of each image slice and $L_{z}=n_{\text {slices }} \Delta z$ is the height of the image stack. This method is inexact, as the number of two-dimensional 
images each particle appears in is not always $2 a / \Delta z$, but we have verified that (2.1) agrees to within $5 \%$ with full three-dimensional methods (Dinsmore et al. 2001). Although it would be desirable to always use three-dimensional methods to compute the volume fraction, (2.1) has the advantage of being applicable to our rapidly flowing samples where three-dimensional imaging is impossible. This method lets us compute the local volume fraction in such samples. Because of local variability and overall uncertainty of the diameters of the particles $(\sim 2 \%)$, we estimate the fractional uncertainties in our measured volume fraction to be of the order of $10 \%$ of the reported values.

In a non-colloidal suspension, it is only the strain undergone by the material which is relevant to the development of the particle fraction and velocity fields (Nott \& Brady 1994), but in a Brownian suspension the flow rate plays a role. The role of the flow rate is captured through the Péclet number, which is the non-dimensional ratio of the time for Brownian diffusion to move a particle its own size, $a^{2} / D_{0}=a^{2} /\left(k T / 6 \pi \eta_{0} a\right)$ compared to $\dot{\gamma}^{-1}$, the characteristic time for shear flow. Thus, the Péclet number is defined as

$$
P e=\frac{6 \pi \eta_{0} \dot{\gamma} a^{3}}{k T},
$$

where $\eta_{0}$ is the viscosity of the solvent, $k$ is the Boltzmann constant, $T$ is the temperature, and $D_{0}=k T / 6 \pi \eta_{0} a$ is the diffusion coefficient of an isolated Brownian particle of radius $a$ in the solvent. In this study we define $\dot{\gamma}=u_{\max } / H$, where $u_{\max }$ is the maximum axial speed of the suspension and $H=25 \mu \mathrm{m}$ is half the width of the channel. There is some temporal variability in the pump system which is reflected in measurements of $u_{\max }$; however, the standard deviation was at most $5 \%$. From the definition it is clear that the Péclet number can be changed in the experiment by adjusting the flow rate, and also by using different sized particles. Thus, decreasing the flow rate is a simple way of increasing the importance of Brownian motion. However, there are experimental limitations: for the $2.3 \mu \mathrm{m}$-diameter particles, values of $P e \lesssim 30$ require flow rates that are at the low end of what the syringe pump can reliably deliver. To access lower values of $P e$, the $1.4 \mu \mathrm{m}$ diameter particles are used: the moderate reduction in particle diameter from $2.3 \mu \mathrm{m}$ to $1.4 \mu \mathrm{m}$ reduces $P e$ by a factor of 4.4 for the same flow rates, thus allowing faster pumping speeds for small $P e$.

For non-Brownian particles, changing the size of the particles (while keeping the channel dimensions unchanged) affects the rate of development of the concentration profile (Hampton et al. 1997) since interactions between larger particles result in larger lateral displacements, relative to the channel size, that drive migration (Phillips et al. 1991). Broadly, larger ratios of $a / H$ result in shorter entrance lengths when the volume fraction is kept constant.

In this investigation, the three adjustable parameters that characterize the flows are the Péclet number, $P e$, the volume fraction, $\phi$, and the ratio $a / H$. Pe quantifies the flow rate by comparing the maximum speed of the particles to Brownian motion, $(2.2) ; \phi$ directly affects the frequency of particle interactions; the ratio of particle size to channel width $a / H$ is expected to influence how quickly particle migration occurs as theory predicts the particle flux to scale as $(a / H)^{2}$.

The flow rate is set by the pump. Locally, the velocity profile is measured by crosscorrelating successive pairs of images in the image stacks. The result, as expected and previously observed (Lyon \& Leal 1998; Frank et al. 2003), is a nearly parabolic profile for axial velocity as a function of $z$ (figure 4, open circles). At larger concentrations $(\phi \gtrsim 0.3)$, there is a significant flattening of the profile near the channel centre, far downstream of the pump. In that case, the concentration in the lower shear 


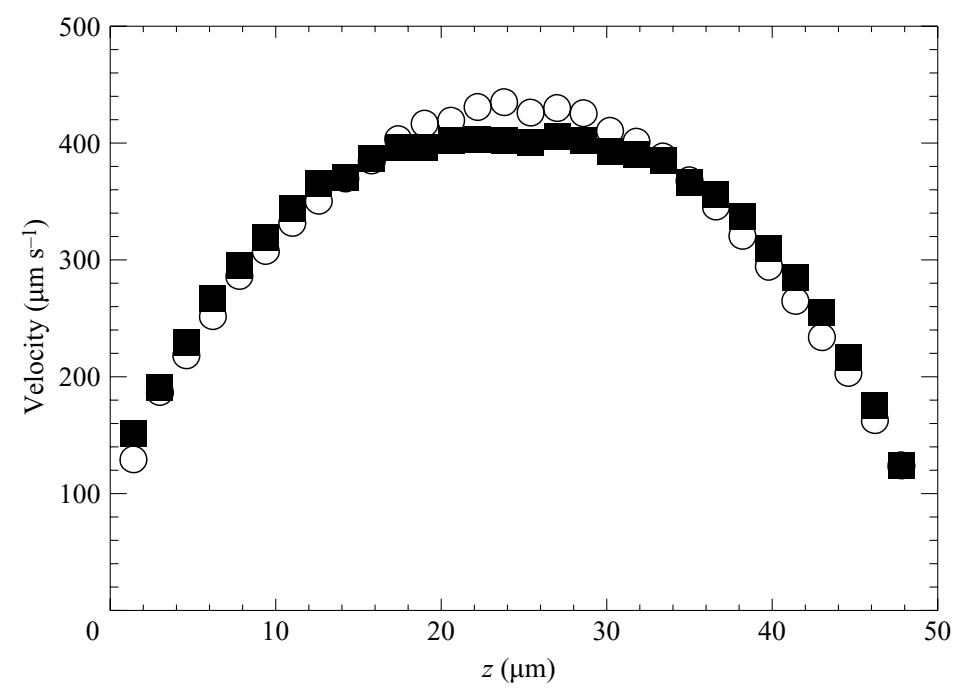

Figure 4. Blunting of velocity for a suspension of $1.4 \mu \mathrm{m}$ diameter particles at $\phi=0.33$ and $P e=60$ (volume flow rate $8.5 \mathrm{nl} \mathrm{s}^{-1}$ ). The nearly parabolic curve formed by the $\bigcirc$ symbols represents the velocity profile at $x / H=20$ while the partially flattened profile formed by the symbols is the profile at $x / H=1040$. For these data, the concentration profile $\phi(x, z)$ reaches $95 \%$ of full development at $x / H=800$.

regions near the centre becomes high enough (from particle migration) that significant enhancement of viscosity occurs, and shearing motion of the dispersion is inhibited. The result is observed as a blunting of the velocity profile as the sample evolves (figure 4, solid squares).

\section{Modelling the evolving flow}

The flow in the channel has been analysed using the approach of Morris \& Boulay (1999) with the influence of Brownian motion on the particle normal stresses included in the constitutive form proposed in Frank et al. (2003). Because the rapid variation in conditions is across the channel (in the $z$-direction in the notation presented in figure 1) while axial evolution is slow, a solution method which neglects the axial variation in order to solve for the velocity at each axial position is used, and the resulting particle stresses are used to predict the variation of the particle fraction with position downstream. This 'marching' method is presented in detail in Miller \& Morris (2006). Specifically, the flow is described by the steady particle conservation equation,

$$
\left\langle u_{x}\right\rangle \frac{\partial \phi}{\partial x}=-\nabla \cdot \boldsymbol{j}_{\perp},
$$

in which the particle flux relative to the bulk motion is given by $\boldsymbol{j}_{\perp}=\phi\left(\langle\boldsymbol{u}\rangle_{P}-\langle\boldsymbol{u}\rangle\right)$, where $\langle\boldsymbol{u}\rangle_{P}$ is the particle phase average velocity and $\langle\boldsymbol{u}\rangle$ is the bulk suspension average velocity, which here is approximated as purely $x$-directed as indicated by the convective derivative on the left-hand side. The neglect of the non-zero $\left\langle u_{z}\right\rangle$ (this bulk cross-stream motion is required by the continuity equation because $\partial u_{x} / \partial x \neq 0$ during development) is considered in Miller \& Morris (2006) and found to have negligible effect on channel-flow development length. The particle flux can be obtained from a 
balance of drag and stress divergence from the particle-phase momentum balance

$$
\boldsymbol{j}_{\perp}=\phi\left(\langle\boldsymbol{u}\rangle_{P}-\langle\boldsymbol{u}\rangle\right)=\frac{2 a^{2}}{9 \eta_{o}} f(\phi) \nabla \cdot \Sigma_{P},
$$

in which $\Sigma_{P}$ is the particle contribution to the stress, and $f(\phi)$ represents the average mobility of the particles, and is taken as the sedimentation hindrance function with form $f(\phi)=(1-\phi)^{\alpha} ; \alpha=2$ is used based on a comparison of modelling (Miller \& Morris 2006) to experimental axially-evolving profiles in tube flow (Hampton et al. 1997).

The particle mass and momentum conservation combined in the form (3.1-3.2) simplify under the assumption that variations of $\Sigma$ are primarily with $z$ to

$$
\left\langle u_{x}\right\rangle \frac{\partial \phi}{\partial x}=-\frac{2 a^{2}}{9 \eta_{o}} \frac{\partial}{\partial z}\left(f(\phi) \frac{\partial \Sigma_{P, z z}}{\partial z}\right) .
$$

In order to obtain $\left\langle u_{x}\right\rangle$, we use the axial momentum equation,

$$
0=-\frac{\mathrm{d} P(x)}{\mathrm{d} x}+\frac{\partial}{\partial z}\left(\eta_{s}(\phi) \frac{\partial\left\langle u_{x}\right\rangle}{\partial z}\right)
$$

where $\mathrm{d} P(x) / \mathrm{d} x$ is the bulk pressure gradient applied to the mixture. From the solution we obtain $\dot{\gamma}=\left|\partial u_{x} / \partial z\right|$ which is required in order to determine the local Pe and thus to specify the particle normal stress

$$
\frac{\Sigma_{P, z z}}{-k T / \frac{4}{3} \pi a^{3}}=a(\phi)+\frac{2 P e}{9}\left[b^{-1}+c^{-1}\right]^{-1},
$$

in the model presented in detail in $\S 3.2$ of Frank et al. (2003). Here, $a(\phi)$ is the dependence of the purely thermal (Brownian) stresses upon $\phi$

$$
a(\phi)=3 \phi\left(1-\frac{\phi}{\phi_{\max }}\right)^{-1}
$$

while $b(\phi, P e)$ and $c(\phi)$ represent the shear-dependent stresses,

$$
b(\phi, P e)=0.7 \operatorname{Pe} \phi\left(1-\frac{\phi}{\phi_{\max }}\right)^{-3}, \quad c(\phi)=0.8 \eta_{n}(\phi),
$$

where $\eta_{n}$ is the normal stress viscosity (Morris \& Boulay 1999) and goes as $\eta_{n} \sim \phi^{2}(1-$ $\left.\phi / \phi_{\max }\right)^{-2}$. We use $\phi_{\max }=0.68$. For large $P e$, the stresses reduce to the term involving $c(\phi)$ and are proportional to $\eta_{0} \dot{\gamma}$ (noting that $k T \times P e \sim \eta_{0} \dot{\gamma}$ ). This simple constitutive law for the normal stresses was developed to capture theoretical limits at low and high $P e$, as well as low and high $\phi$, and to fit the $P e$-dependence of normal stress differences (largely from simulation: Phung, Brady \& Bossis 1996) as well as particle pressure (Jeffrey, Morris \& Brady 1993), as discussed in Frank et al. (2003). Unfortunately, sufficient volume of the colloidal dispersion was not available to perform rheometric experiments on our materials.

Note that in this formulation, gradient diffusion, whether due to Brownian motion or shear-induced, arises naturally, as a term proportional to $\nabla \phi$ is obtained directly,

$$
\boldsymbol{j}=\frac{2 a^{2}}{9 \eta_{o}} f(\phi)\left[\frac{\partial \Sigma_{P}}{\partial \phi} \cdot \nabla \phi+\frac{\partial \Sigma_{P}}{\partial \dot{\gamma}} \cdot \nabla \dot{\gamma}\right],
$$

and for the compressive normal stresses given by (3.5), $\Sigma_{P}$ provides the expected negative sign in the leading term of the brackets on the right-hand side - i.e. 


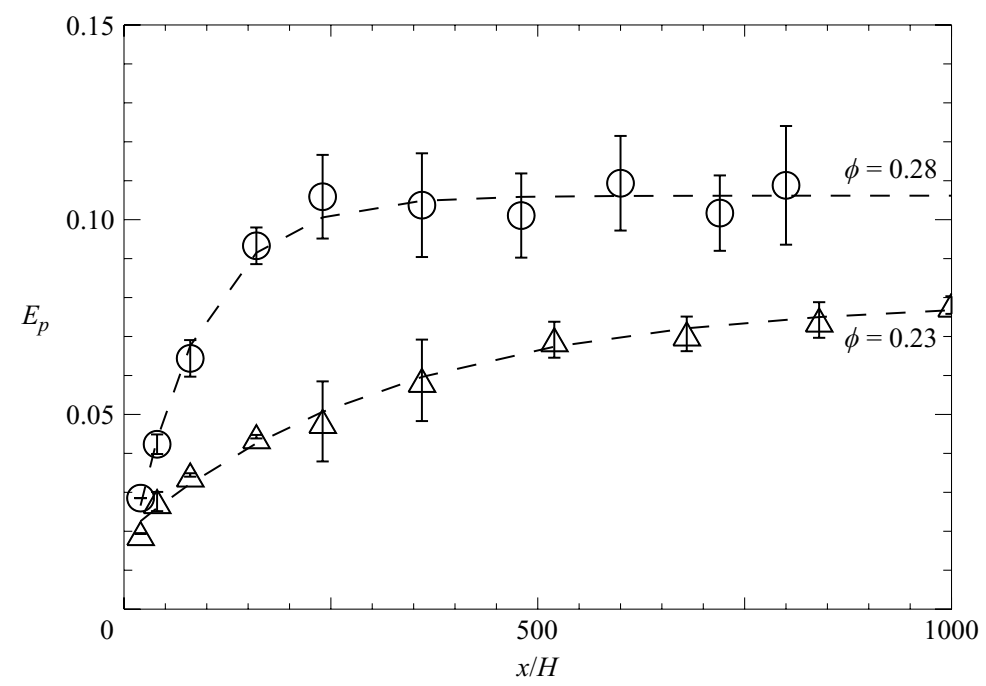

FIGURE 5. Development of the inhomogeneous concentration profile for suspensions of $2.3 \mu \mathrm{m}$ diameter particles at two different volume fractions at $P e=80$ (volume flow rate $1.7 \mathrm{nl} \mathrm{s}^{-1}$ ). $E_{p}$ characterizes the flow development (see (4.1)). The distance $x$ from the channel inlet is non-dimensionalized by the half-width $H$ of the channel. The dashed lines are fits using (4.2).

compressive, or dispersive, normal stresses act to dissipate gradients. The second term in the brackets results from the shear-rate dependence and therefore may generate a gradient in $\phi$; this is the essential driving force for migration. This term would have a more complicated form if the full dependence of $\Sigma_{P}$ on the velocity gradient tensor (here expressed simply through $\dot{\gamma}$ ) were used.

\section{Results}

The development length of the concentration profile is quantified following a method similar to that defined by Hampton et al. (1997). A scalar measure of the development is given by an evolution parameter $E_{p}$ which for a local concentration $\phi$ is given by

$$
E_{p}(x)=\frac{1}{2 H} \int_{0}^{2 H}\left|\frac{\phi(x, z)}{\langle\phi(x, z)\rangle_{z}}-\frac{\phi_{r e f}(z)}{\left\langle\phi_{r e f}(z)\right\rangle_{z}}\right| \mathrm{d} z,
$$

where $\phi_{r e f}(z) \equiv \phi(x=0, z)$ is the volume fraction profile at the inlet, $\langle\phi(x, z)\rangle_{z}$ is the local cross-sectional average volume fraction, and $2 H$ is the height of the channel. Thus, $E_{p}(x)$ is zero for a suspension with uniform concentration over a cross-section, and grows with $x$ from the inlet as the initially uniform suspension becomes nonuniform owing to particle migration.

Equation (4.1) hints that the cross-sectional average volume fraction $\langle\phi(x, z)\rangle_{z}$ is not constant - it decreases with $x$. Downstream, particle migration towards the fasterflowing centre means that, to maintain the same total particle flux as the inlet where $\phi(x=0, z)$ is uniform, $\langle\phi(x, z)\rangle_{z}$ must decrease.

As the concentration profile develops downstream, a plot of $E_{p}$ versus distance from the channel inlet shows that the concentration profile asymptotically approaches a constant value (figure 5). The rate of growth of $E_{p}$ with $x$ is characterized by fitting it to an exponential function (dashed line in figure 5) from which an entrance length 

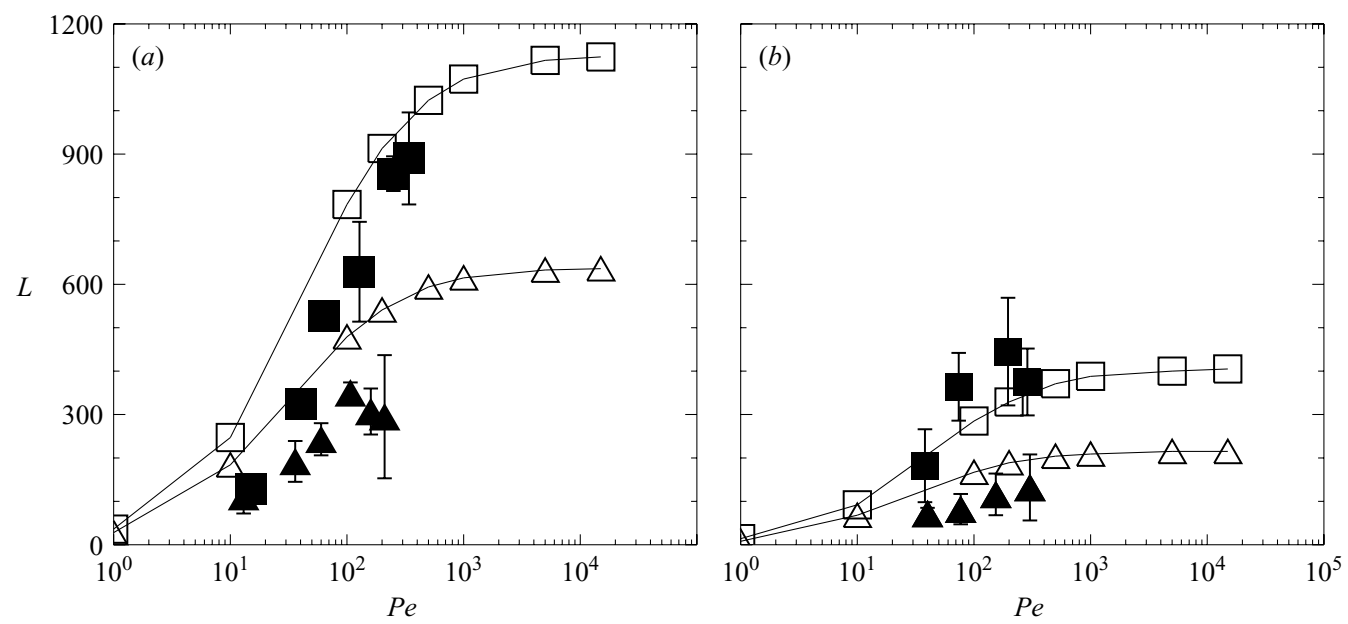

FiguRE 6. Plots of entrance length as a function of $P e$ showing results for both experiment (solid symbols) and computation (open symbols). (a) Results for $1.4 \mu \mathrm{m}$ diameter particles. (a) Experimental data: $\mathbf{\square}, \phi=0.24 ; \boldsymbol{\Delta}, \phi=0.35$. Model data: $\square, \phi=0.24 ; \Delta, \phi=0.35$. Experimental data at $\phi=0.31$ is similar to that for $\phi=0.35$ and is not shown. $(b)$ Results for $2.3 \mu \mathrm{m}$ diameter particles. Experimental data: $\boldsymbol{\square}, \phi=0.24 ; \boldsymbol{\Delta}, \phi=0.28$. Model data: $\square$, $\phi=0.24 ; \Delta, \phi=0.35$. Experimental data at $\phi=0.37$ is similar to that for $\phi=0.28$ and is not shown. The vertical bars represent the standard deviation of the range of measured entrance lengths.

$L$ is obtained. The exponential fit reduces experimental noise and is of the form

$$
E_{p}^{F i t}(X)=\alpha_{1}\left(1-\mathrm{e}^{-X / L}\right)+\alpha_{2},
$$

with $X=x / H$ the dimensionless distance from the inlet; $\alpha_{1}, \alpha_{2}$ and $L$ are fitting parameters. We define parameter $L$ to be the entrance length, which, in our experiments, is usually $O(100)$ half-channel widths. The evolution parameter $E_{p}$ typically reaches $95 \%$ of its asymptotic value at distances $O(1000)$ half-channel widths downstream from the inlet. The $\alpha_{2}$ offset parameter is necessary since the evolution parameter $E_{p}$ is a difference of inherently noisy local volume fraction measurements along the channel that cannot completely cancel out even if they are taken at the same axial position. The fitting form of (4.2) is a slight variation of that suggested by Hampton et al. (1997) where the function is taken as

$$
E_{p}^{F i t}(X)=\alpha_{1}\left(1-\exp \left(\alpha_{2} X^{0.8}\right)\right)+\alpha_{3},
$$

and the entrance length is defined as the distance from the channel inlet for which the evolution parameter $E_{p}$ reaches $95 \%$ of its asymptotic value. Although the two methods yield similar results, the entrance length definition of (4.2) is used here.

The entrance length is measured for several different values of $P e$ with the large $(2.3 \mu \mathrm{m}$ diameter $)$ and small $(1.4 \mu \mathrm{m}$ diameter $)$ particles. All data points for the plots of $E_{p} v$ s. $x / H$ (for example, figure 5) are obtained from performing at least two experiments, and most data points are determined from three experiments; furthermore, for each axial position, $x / H$, at least two stacks of images are taken per experiment. The entrance lengths for different $\phi$, as a function of $P e$, are shown in figure 6 . The error bars represent the standard deviation of the range of measured entrance lengths. 
As $P e$ increases, the entrance length $L$ increases, eventually reaching an apparent plateau for $P e \gtrsim 100$ (figure $6 a$ ). This is reasonable; for $P e \ll 100$, Brownian motion is most significant and thus has a strong influence on the behaviour. For large $P e$, Brownian motion is less important and the entrance length approaches a constant value; at that point, it is entirely due to hydrodynamic effects which dominate the thermal effects. The transition region, $P e \sim 100$, is approximately the same for both particle sizes (figure 6) and different volume fractions, indicating that $P e$ captures the relevant physics. (This is least obvious for the solid triangles in figure 6, corresponding to the larger particles and a higher volume fraction; these data have large scatter which prevents them from supporting this conclusion. We have also taken data at $\phi=0.37$ which looks both qualitatively and quantitatively similar to the $\phi=0.28$ data.)

A second expected result is that the larger particles have shorter overall entrance lengths (figure $6 b$ ). This agrees with previous experiments with non-Brownian particles, which found shorter entrance lengths for larger $a / R$ (the ratio of particle size and cylindrical tube radius), (Hampton et al. 1997). In other words, the effect of particle size is reflected in the concentration profile at each $x$ : shear-induced particle migration of large particles is more rapid than that of small ones since interparticle interactions lead to bigger cross-stream displacements for larger particles (Phillips et al. 1991). For our particle sizes, the relevant aspect ratios are $a / H=0.028$ and $a / H=0.046$.

The predictions from the model are qualitatively similar to the results of the experiments (figure 6). There is strong entrance-length dependence on $\mathrm{Pe}$ at small $\mathrm{Pe}$, whereas for $P e=O(100)$, significantly weaker $P e$ influence is observed; the transition from small to large $P e$ for the $\phi=0.24$ and 0.35 dispersions occurs from $P e=10$ to 100 . Quantitatively, the agreement with experiment is roughly within $25 \%$ for the lower volume fraction $\phi=0.24$ data and within $50 \%$ for the higher volume fraction data. The model predicts that the entrance length continues to grow (albeit much more slowly) over several decades in $P e$.

A third result shown in figure $6(a, b)$ is the volume-fraction dependence: for a fixed particle size and $P e$, the higher volume-fraction data show a shorter entrance length $L$. To further illustrate this, figure 7 shows a plot of experimental data and model predictions for entrance-length dependence on bulk volume fraction. We show results for $2.3 \mu \mathrm{m}$ diameter particles at $P e=150$ (both experiment and model), as well as additional model predictions at infinite $P e$. The decreasing entrance length is a result of the migration being due to particle interactions which become stronger at elevated $\phi$. It has been noted that migration should become nearly instantaneous as $\phi \rightarrow \phi_{\max }$ (Morris \& Brady 1998; Miller \& Morris 2006) and experiments at extremely concentrated conditions appear to confirm this for non-colloidal suspensions (Ovarlez, Bertrand \& Rodts 2006). Figure 7 also demonstrates that the entrance length is nearly constant for $P e>100$, given the close agreement in the model data between $P e=150$ and $P e=\infty$. Quantitatively, the $P e=150$ experimental data are close to the model predictions and show a reduced entrance length with increasing $\phi$, but with the reductions becoming small as $\phi$ becomes large. This is noted in figure 6 where plots of the two higher volume fractions have been omitted because of similar entrance lengths.

\section{Discussion}

The key result of this study is that for pressure-driven flows of Brownian suspensions, Brownian motion affects the evolution, and correspondingly the entrance 


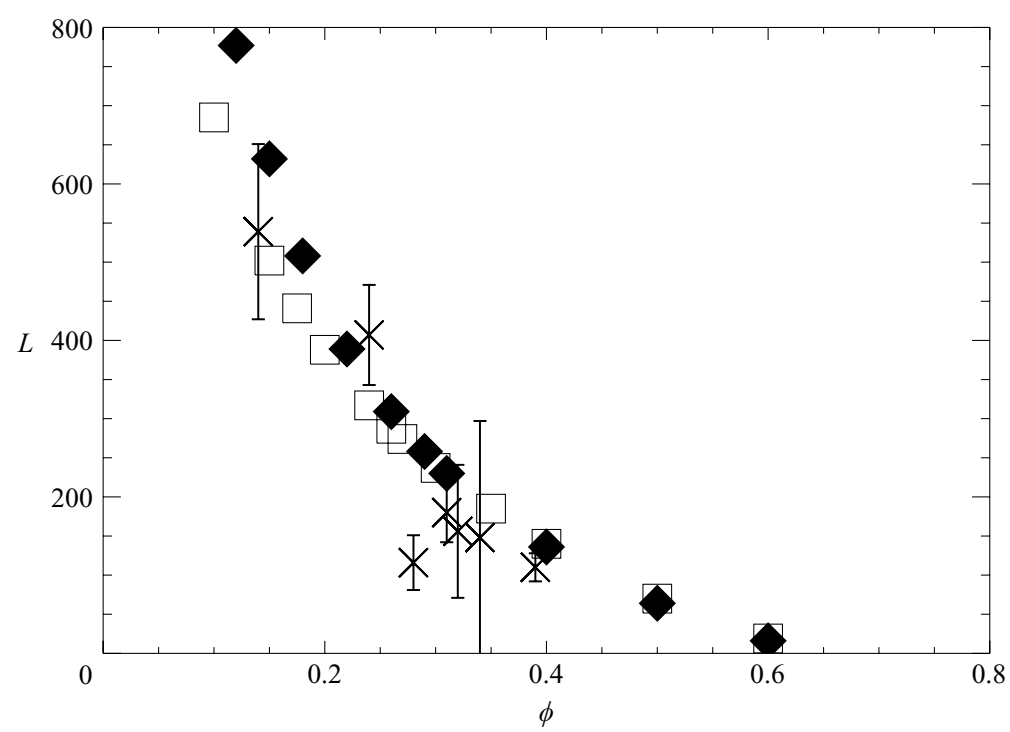

FIGURE 7. Entrance length vs. $\phi . \times$, obtained experimentally using $2.3 \mu \mathrm{m}$ diameter colloidal suspensions with $P e=150$ (volume flow rate $3.3 \mathrm{nl} \mathrm{s}^{-1}$ ); $\square, \diamond$, corresponding numerical analyses with $P e=150$ and $P e=\infty$, respectively. The vertical bars represent the standard deviation of the range of measured entrance lengths.

length, of the cross-stream concentration profile. When $P e \ll 100$, the entrance length is found to have a significant dependence on the flow rate. This contrasts with nonBrownian suspensions which do not have a dependence of entrance length on flow rate - experiments by Hampton et al. (1997) showed that when $O(1000) \mu \mathrm{m}$ diameter particles are pumped through a cylinder, the cross-stream concentration profiles are unchanged when the pumping rates are changed, thereby precluding any entrancelength dependence of the concentration profile on flow rate. As noted above, this result is consistent with theories that predict that shear-induced particle migration depends only on the total strain for low-Reynolds-number flows (Phillips et al. 1991). The independence of entrance lengths on rate for non-Brownian suspensions is simply understood as being a result of the linearity of the hydrodynamic equations, and the fact there is no rate in the problem other than the imposed flow rate. Stated in other words, a higher flow rate (larger $\left\langle u_{x}\right\rangle$ ) leads to a migration scaling linearly as $\left\langle u_{x}\right\rangle$, but likewise the distance travelled downstream in a given time scales linearly with this velocity, so there is no effect upon the entrance length, a theoretical prediction confirmed by experiments (Hampton et al. 1997; Lyon \& Leal 1998).

The dependence of the fully developed concentration profile on flow rate for Brownian suspensions helps to explain the flow rate- or $P e$-dependence of the entrance length. Since the steady fully developed concentration profile results from a balance between shear- and Brownian-driven stresses, $P e$ sets the extent of variation of the fully developed profile, i.e. it fixes the maximum value, $E_{p}(\infty)$, of the evolution parameter. However, the actual approach to $E_{p}(\infty)$ is to a good approximation still determined by the total strain. This is illustrated by figure $8(a)$ where the evolution parameters for experiments with $P e=15$ and $P e=70$ track relatively closely together initially. For a significant distance from the inlet, the lower and higher $P e$ profiles have approximately equal values of $E_{p}$ (migration). Large differences appear only as the lower $P e$ curve approaches its maximum $E_{p}$. Figure $8(b)$ shows that this is 

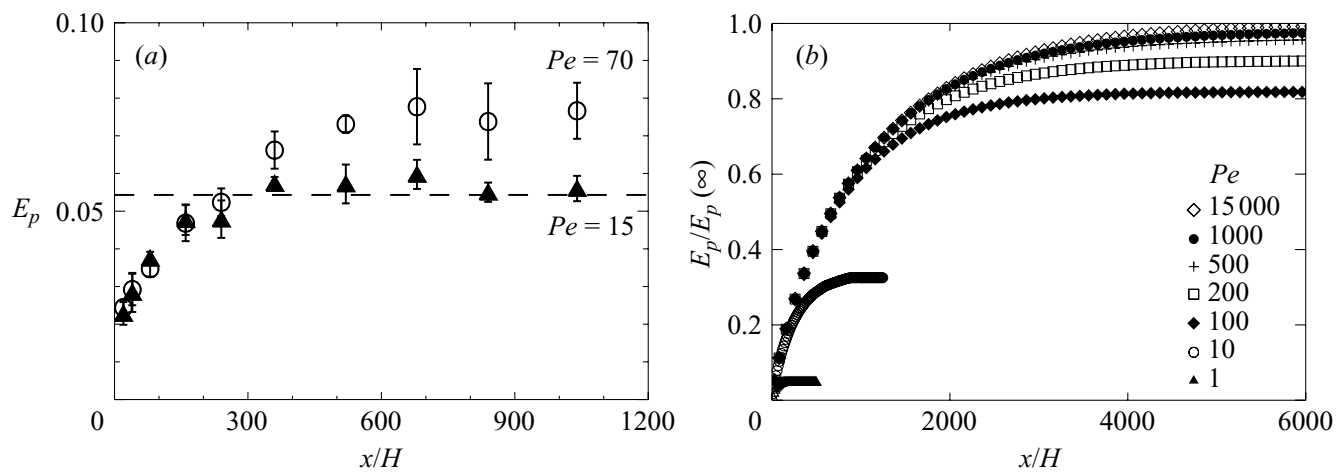

FIGURE 8. Experimental and model plots of evolution parameter vs. distance from the inlet. (a) Experiment results for a suspension of $1.4 \mu \mathrm{m}$ diameter particles at $\phi=0.35$, with $\mathrm{Pe}$ as indicated. The horizontal dashed line is $95 \%$ of the maximum $E_{p}$ for the $P e=15$ data. The vertical bars represent the standard deviation of the range of parameter $E_{p}$ for several different experiments. $(b)$ Model results for a large range of $P e . E_{p}$ has been normalized by the asymptotic value at infinite $P e$.

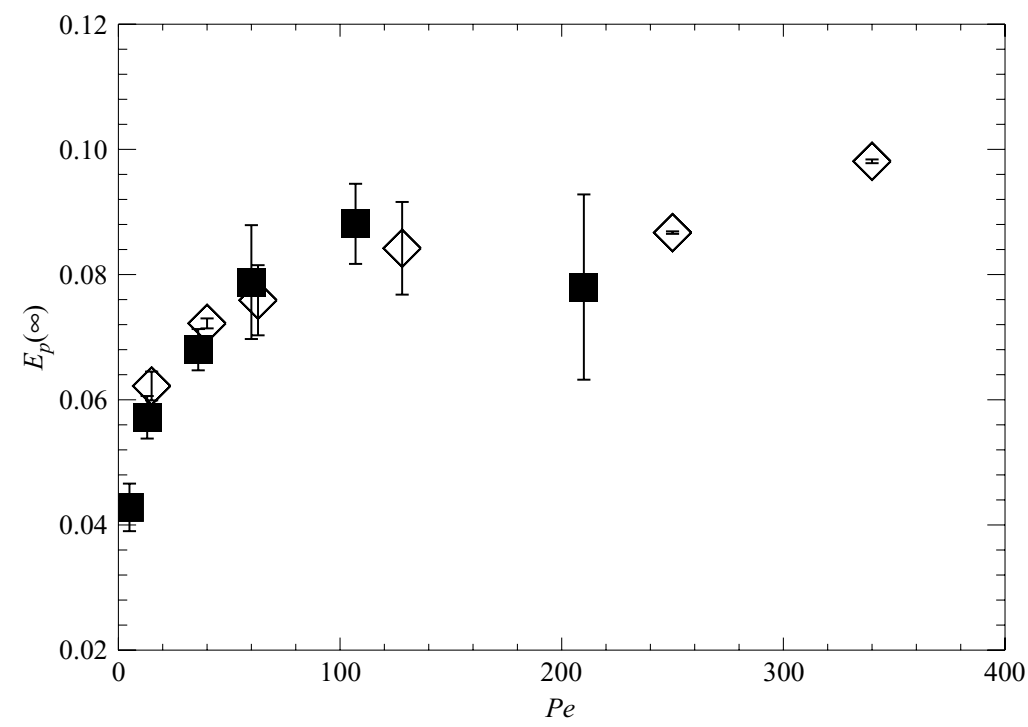

FIGURE 9. Experimental data of the asymptotic value of evolution parameter vs. Pe for a suspension of $1.4 \mu \mathrm{m}$ diameter particles at $\phi=0.24(\diamond)$ and $\phi=0.28$

precisely as predicted in the model solution; in this figure, $E_{p}$ is shown normalized by its asymptotic value at infinite $P e$. We observe that where $E_{p}(x)$ is far from $E_{p}(\infty)$, the lower $P e$ flows have approximately the same evolution with $x$ in their concentration profile as the higher $P e$ flows. Additionally, figure $8(b)$ along with figure 9 show that the asymptotic value of the evolution parameter, $E_{p}(\infty)$, indeed increases with $P e$. Consistent with observations of the entrance length, figure 9 shows that $E_{p}(\infty)$ grows significantly with increasing $P e$ until $P e \approx O(100)$, at which point $E_{p}(\infty)$ begins to saturate. This is reflected in the computations of figure $8(b)$ which also show that increases in $P e$ from $P e=100$ to $P e=15000$ result in only a $20 \%$ increase in $E_{p}$. Thus, it appears that for Brownian suspensions the entrance length increases with $P e$ because of the increase in total particle migration with flow rate. 

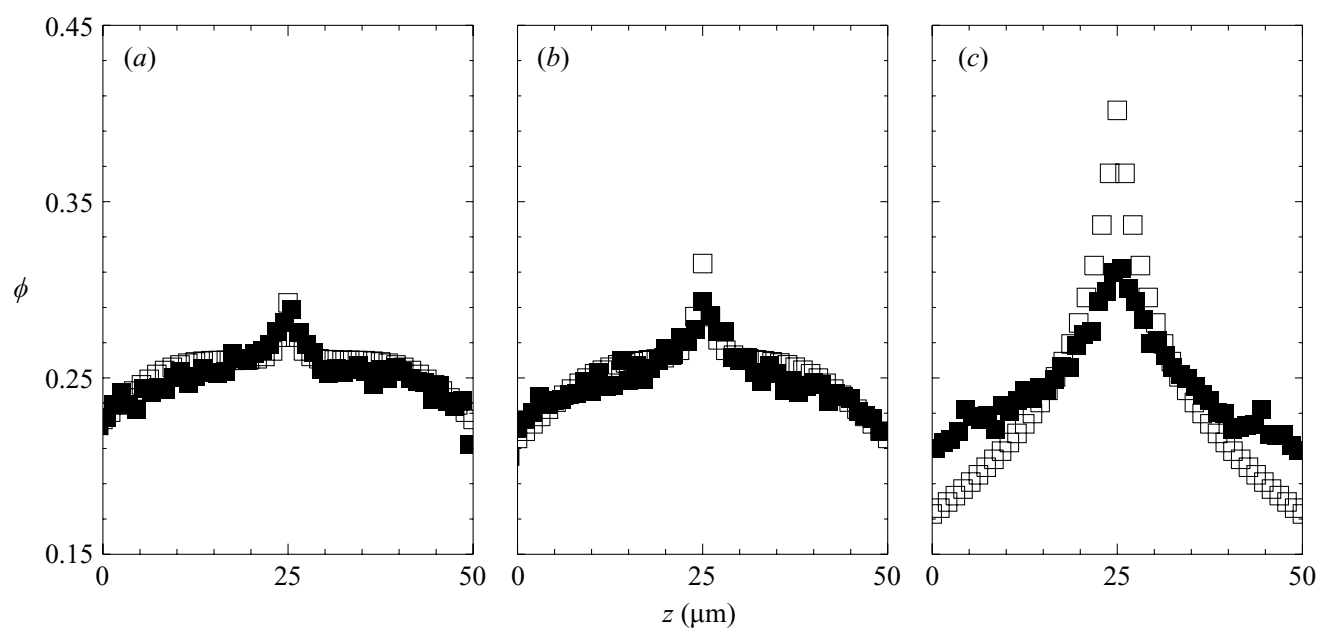

FiguRE 10. Comparison between experimental ( $\square)$ and model $(\square)$ volume fraction profiles at three intervals along the channel. The suspension is of $1.4 \mu \mathrm{m}$ diameter particles at volume fraction $\phi=0.26$ and $P e=129$ (volume flow rate $17 \mathrm{nl} \mathrm{s}^{-1}$ ). (a) Near the entrance at $x / H=80$, the model is in good agreement with the experiment. (b) Farther downstream at $x / H=160$, differences in the extent of particle migration begin to appear, and (c) at $x / H=1360$, the profiles are significantly different. The profiles reach $95 \%$ of full development at $x / H=1990$ for the experiment and $x / H=2250$ for the model.

While the model captures the essential results of the experiment, quantitative differences are found in the detailed $\phi(x, z)$. As noted in Frank et al. (2003), the model does not reproduce exactly the predicted concentration profiles at full development. Figure 10 shows good agreement between the experimental data and the model at short distances from the inlet. However, farther along the channel the model overestimates the particle migration. Nevertheless, the characteristic development lengths of the model and experiment are comparable. One factor which was noted (Frank et al. 2003) to contribute to the lack of agreement in the profiles is the application of the rheological model at arbitrarily small scales, when the rheology of the material should properly be employed only in volumes containing several particles. Here, we have used the approach of Miller \& Morris (2006), who addressed this issue for non-colloidal suspensions by using a non-local stress contribution in which the stress depends upon the shear rate averaged about the point of interest in a finite volume. Agreement with the Hampton et al. (1997) results for $\phi(r)$ in tube flow of concentrated suspensions, is improved when the shear rate is averaged over a volume of the order of the size of the particle, but we note that the appropriate form to use for a colloidal suspension remains uncertain. A second factor which may contribute is the use at low solids fraction of a model designed for highly concentrated suspensions, where normal stress is comparable to shear stress $(\phi \geqslant 0.35$, Morris \& Boulay 1999). A third factor could be the charge on the particles. The modelling assumes the particles are Brownian hard spheres, but the charge causes an electrostatic repulsion between the particles that may be sufficiently strong to account for some of the deviation between the measurements and the model. Intuitively, it might be expected that the effect of the repulsion would be to suppress migration as particles in the centre become more concentrated, hence the good agreement at short distances down the channel, but poor agreement farther downstream. 
Migration theories, such as those of Nott \& Brady (1994) and Phillips et al. (1991), predict an axial profile development length inversely proportional to $(a / R)^{2}$, where $a$ is the particle radius and $R$ is the radius of the cylindrical tube that they flow through. However, experimental data by Hampton et al. (1997) shows a range of values for the exponent, increasing linearly from 0.4 for $\phi=0.2$ to 1.8 for $\phi=0.45$. They speculate that this may be due to discrete particle-size effects - their experiments use particles for which $a / R$ is as large as 0.0625 , probably above the size for which the continuum approach is strictly valid (say 0.02 ). For this study, the ratios are $a / H=0.046$ and $a / H=0.028$. Determining the exponent in our experiments by plotting $L \times(a / H)^{n} v$ s. $P e$ and adjusting $n$ until the curves of similar volume fractions collapse onto one another, values $n \approx 1$ for $\phi=0.24$ and $n \approx 2$ for $\phi=0.35$ are obtained. That is, even for our experiments where discrete particle-size effects are smaller than those of Hampton et al. (1997), there is a wide range of values of the exponent. The exponents are slightly closer to that of the migration theories, but still show a strong $\phi$ dependence. The scaling for the model ( $(3)$ is closer to the theoretical value of $n=2$, although it is not constant either. For the suspensions with solids fraction $\phi=0.24$, the plots for ratios $a / H=0.028$ and $a / H=0.046$ collapse onto each other when $n=2.05$, whereas for the $\phi=0.35$ suspensions, the plots collapse when $n=2$.2. It would be instructive to repeat this work with particles that are small enough to be below the $a / H=0.02$ continuum limit and determine whether the exponent still has a volume-fraction dependence.

\section{Conclusions}

We find significant dependence of entrance length on Péclet number for flows with $P e \ll 100$. The effect of Brownian motion on shear-induced particle migration is to reduce the axial distance required for a Brownian suspension to reach its fully developed inhomogeneous concentration profile. It does so by suppressing the total migration. As $P e$ reaches $O(100)$, the Brownian effect becomes weak compared to the shear-dependent stresses that cause migration and the entrance length correspondingly becomes a weaker function of $P e$.

The modelling based upon a two-fluid analysis, in which particle migration results from spatial variation of the suspension normal stresses, successfully predicts the observed trends with $P e$ and $\phi$ using the constitutive modelling proposed in Frank et al. (2003). In particular, the pronounced increase in entrance length as $P e$ grows to $O(100)$ in the moderately concentrated suspensions agrees with experiment. The model predictions are in complete agreement with the somewhat surprising experimental observation that the evolution, as characterized by $E_{p}$, follows a similar trend in its growth with distance downstream for all $P e$ in the early stages (small axial distance $x / H)$ with levelling at lower ultimate values, $E_{p}(\infty)$, and hence at smaller $x / H$ for smaller $\mathrm{Pe}$. The work thus provides firm guidance for prediction of the evolution scales in pressure-driven flows of colloidal dispersions.

This material is based upon work supported by the National Science Foundation under Grant 0239109.

\section{REFERENCES}

Antl, L., Goodwin, J. W., Hill, R. D., Ottewill, R. H., Owens, S. M., Papworth, S. \& Waters, J. A. 1986 The preparation of polymethyl methacrylate latices in non-aqueous media. Colloids Surfaces 17, 67. 
Dinsmore, A. D., Weeks, E. R., Prasad, V., Levitt, A. C. \& Weitz, D. A. 2001 Three-dimensional confocal microscopy of colloids. Appl. Optics 40, 4152.

Fournier, R. L. 1999 Basic Transport Phenomena in Biomedical Engineering. Taylor \& Francis.

Frank, M., Anderson, D., Weeks, E. R. \& Morris, J. F. 2003 Particle migration in pressure-driven flow of a Brownian suspension. J. Fluid Mech. 493, 36.

Hampton, R. E., Mammoli, A. A., Graham, A. L. \& Tetlow, N. 1997 Migration of particles undergoing pressure-driven flow in a circular conduit. J. Rheol. 41, 621.

JefFrey, D. J., Morris, J. F. \& Brady, J. F. 1993 The pressure moments for two rigid spheres in low-Reynolds-number flow. Phys. Fluids A 5, 2317.

Koh, C. J., Hookham, P. \& Leal, L. G. 1994 An experimental investigation of concentrated suspension flows in a rectangular channel. J. Fluid Mech. 266, 1.

Leighton, D. T. \& Acrivos, A. 1987 The shear-induced migration of particles in concentrated suspensions. J. Fluid Mech. 181, 415.

Lyon, M. K. \& LeAL, L. G. 1998 An experimental study of the motion of concentrated suspensions in two-dimensional channel flow. Part 1. Monodisperse systems. J. Fluid Mech. 363, 25.

Miller, R. M. \& Morris, J. F. 2006 Normal stress-driven migration and axial development in pressure-driven flow of concentrated suspensions. J. Non-Newtonian Fluid Mech. 135, 149.

Morris, J. F. \& Boulay, F. 1999 Curvilinear flows of noncolloidal suspensions: the role of normal stresses. J. Rheol. 43, 1213.

Morris, J. F. \& Brady, J. F. 1998 Pressure-driven flow of a suspension: buoyancy effects. Intl J. Multiphase Flow, 24, 105.

Nott, P. R. \& Brady, J. F. 1994 Pressure-driven suspension flow: simulation and theory. J. Fluid Mech. 275, 157.

Ovarlez, G., Bertrand, F. \& Rodts, S. 2006 Local determination of the constitutive law of a dense suspension of noncolloidal particles through magnetic resonance imaging. J. Rheol. 50, 259.

Phillips, R. J., Armstrong, R. C., Brown, R. A., Graham, A. L. \& Авbott, J. R. 1991 A constitutive equation for concentrated suspensions that accounts for shear-induced particle migration. Phys. Fluids 4, 30.

Phung, T. N., Brady, J. F. \& Bossis, G. 1996 Stokesian dynamics simulation of Brownian suspensions. J. Fluid Mech. 313, 181. 